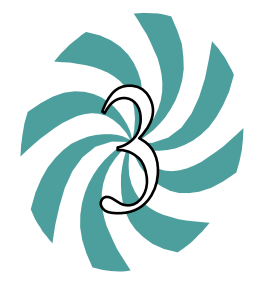

Tecnociencia, Vol. 22, $\mathrm{N}^{\circ}$ 1: 37-47

enero-junio 2020

\title{
MATRIZ DE SÚPER TELEFOTOS PARA LA DETECCIÓN DE OBJETOS ASTRONÓMICOS DE BAJO BRILLO SUPER- FICIAL EN CHURUQUITA CHIQUITA DE COCLÉ, PANAMÁ
}

\author{
1,2Pablo Weigandt \& ${ }^{2}$ Daniel Weigandt \\ ${ }^{1}$ Universidad de Panamá. Email: pablo@weigandt.net \\ ${ }^{2}$ Centro de Investigación de Física Aplicada - Universidad Autónoma de Chiriquí \\ Email: daniel@weigandt.net
}

\section{RESUMEN}

Se describe la construcción del "Sistema novedoso de óptica refractiva integrada" (SNORRI). Este es un sistema de cuatro súper teleobjetivos alineados junto con cuatro cámaras CCD de última generación, integradas en una red. Softwares comerciales se utilizan para manejar el equipo, integrados en un centro de control de interfaz personalizado.

Este equipo astronómico fue construido en Churuquita Chiquita en el distrito de Penonomé de la provincia de Coclé en la República de Panamá. Su objetivo principal es la detección de estructuras de bajo brillo superficial u objetos tenues en el cielo con un amplio campo de visión. Se discute la buena calidad del sistema y algunos resultados preliminares.

\section{PALABRAS CLAVES}

Instrumentación, astronómica, evolución galáctica, telescopios, procesamiento de imágenes astronómicas. 


\title{
SUPER TELEFOTO ARRAY FOR THE DETECTION OF LOW SURFACE BRIGHNESS ASTRONOMICAL OBJECTS IN CHURUQUITA CHIQUITA OF COCLÉ, PANAMA
}

\begin{abstract}
The construction of the "Novel System of Integrated Refractive Optics" (SNORRI) is described. This is a four aligned super telephoto array system coupled with four last generation CCD cameras integrated in a network. Commercial software are used to drive the equipment that is integrated in a custom made interface control center.

This astronomical equipment was built in Churuquita Chiquita in Penonomé District of Coclé Province in the Republic of Panama. Its primary goal is the detection of low surface brightness structures or faint objects in the sky with a wide field of view. The good quality of the system and some preliminary results are discussed.
\end{abstract}

\section{KEYWORDS}

astronomical instrumentation, galaxy evolution, telescopes, astronomical image processing.

\section{INTRODUCCIÓN}

Como parte de las contribuciones al estudio de la evolución del Universo, es importante detectar las llamadas "estructuras faltantes" y, en particular, los escombros o "debris" en galaxias espirales cercanas (James Bullock \& Kathryn Johnston, 2005; Cooper et al., 2010) en un número estadísticamente significativo. Lamentablemente, por el poco brillo superficial de estas estructuras, es muy difícil su detección debido a la baja relación señal/ruido y por los prolongados tiempos de exposición que necesitan los telescopios convencionales, aunque se han realizado algunas mediciones importantes en los últimos años (Martínez-Delgado et al., 2010; Martínez-Delgado et al., 2015).

Recientemente se han hecho esfuerzos significativos para mejorar esta situación creando instrumentos más eficientes para la detección de objetos astronómicos de muy bajo brillo superficial, como es el caso del dispositivo "Dragonfly telefoto array" (Abraham \& Van Dokkum, 2014) con el cual se han realizado estudios (Van Dokkum et al., 2014). 
Lo anterior motivó la construcción de este sistema astronómico para la detección de objetos de bajo brillo superficial, financiado por la Secretaría Nacional de Ciencia, Tecnología e Innovación (SENACYT) de la Rep. de Panamá, a través del proyecto FID16-010, favorecido en convocatoria pública para el fomento de la investigación y desarrollo.

\section{MATERIALES Y MÉTODOS}

Primeramente, se determinó el lugar propicio para la construcción de este sistema de observación astronómica que se ha dado a conocer como SNORRI ("Sistema Novedoso de Óptica Refractiva Integrada"). Luego de un análisis sobre la contaminación lumínica, la nubosidad, y seguridad del equipo, entre otros factores, se optó por instalarlo en la Comunidad de Churuquita Chiquita, en el Distrito de Penonomé, Provincia de Coclé, República de Panamá ( $8^{\circ} 34^{\prime} 12^{\prime \prime}$ latitud norte, $80^{\circ} 16^{\prime} 48^{\prime \prime}$ longitud oeste).

Se procedió con el ensamblaje, armado de soporte, montaje de lentes y cámaras e instalación de software. Preliminarmente se armó el domo astronómico previa construcción de un piso y fundaciones adecuadas y se instaló la base para la montura robótica. Se hicieron todas las instalaciones de cableado con las computadoras, monitor y demás periféricos. La Matriz de Súper Telefoto quedó debidamente instalada.

La matriz la forman cuatro lentes súper telefoto de marca Canon modelo EF $400 \mathrm{~mm} \mathrm{f/2.8L} \mathrm{IS} \mathrm{II} \mathrm{USM} \mathrm{(Canon} \mathrm{Inc.,} \mathrm{2011)} \mathrm{siendo} \mathrm{éstos}$ extremadamente "rápidos" y contando con lo último en revestimiento antirreflectante ("super spectra coating") lo que los hace ideales para el propósito del proyecto. Estos lentes se montaron en una estructura metálica de fabricación casera quedando alineados para observar una misma región en el firmamento.

El domo observatorio es de la marca NexDome, y las cámaras CCD son modelo Aluma con un CCD KAF-8300 de SBIG - Diffraction Limited de última generación (tercera) que acaba de salir al mercado (Diffraction Limited, 2019). Las cámaras se conectaron a una red de mini computadoras marca Gigabytes Brix, manejada a través de una terminal única. 


\section{RESULTADOS Y DISCUSIÓN}

Se cuenta con el equipo completo y funcionando. Su constitución principal son los cuatro lentes súper telefoto con sus respectivas cámaras CCD montadas en una única estructura de metal laminado de perfil "L" de 2,54 cm, unida a la montura robótica Paramount MX+ que supera con creces la capacidad para manipular los aproximadamente 20kg de la Matriz (Software Bisque, 2015) y que a su vez está sujeta a la base fija en tierra. El sistema computacional está armado con el hardware y sofware más apropiado. Todo lo anterior está dentro del domo astronómico debidamente instalado.

Cada uno de los cuatro lentes se fijó en una distancia focal de $400 \mathrm{~mm}$ y cuentan con una apertura de $143 \mathrm{~mm}$ lo que ofrece en conjunto una relación focal de f/1.4. El campo de visión es de $2,6^{\circ} \times 1,9^{\circ}$. En las figuras 1 y 2 se observa el sistema.

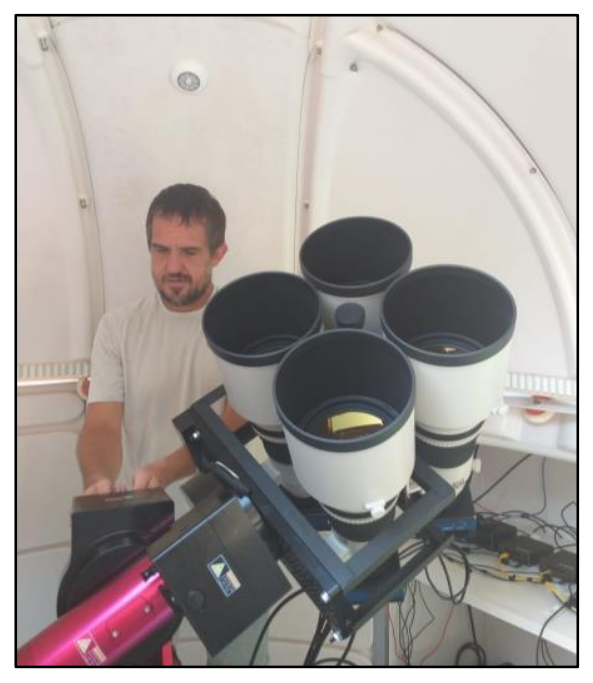

Fig. 1 La estructura de hierro sostiene los cuatro lentes súper telefoto. Ésta está sujeta a la montura robótica. A la derecha se observan las cuatro mini computadoras que controlan las cámaras CCD 


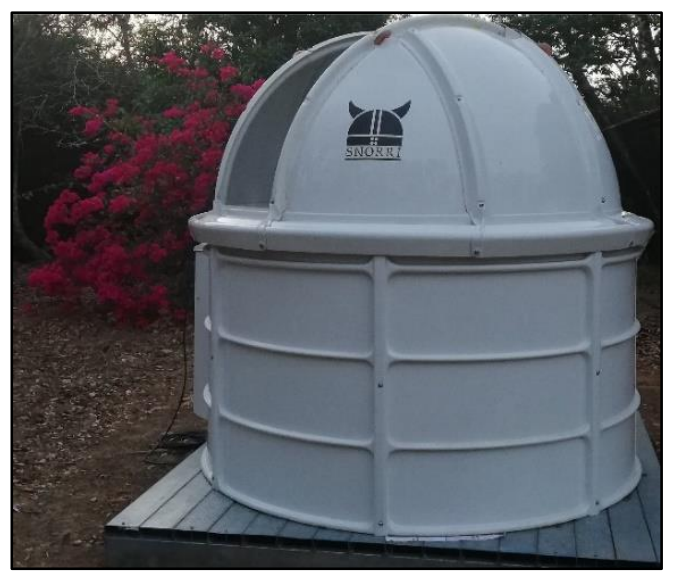

Fig. 2 El Domo Astronómico que resguarda la matriz de súper telefotos

Para el comando central de operaciones, se desarrolló una interface de control central denominada "SNORRI Interface Control Center" (SICC).

SICC es el programa que centraliza las operaciones de SNORRI que se dan a través de la red de computadoras y cámaras que constituyen el sistema informático. Éste consta actualmente de cuatro cámaras y cuatro computadoras conectadas en red LAN con un Switch y que se comunican con SICC a través de TCP. Cada computadora tiene instalado un sistema operativos Windows 7 Professional y MaxIm DL 6.x (Diffraction Limited. 2019).

SICC es un conjunto de tres programas escritos en C\#: SICC, MMDL (Monitor de Maxim DL) y AMDL (Acciones para Maxim DL). El primero está instalado en la llamada "computadora maestra" o "Master PC" que maneja el operador, y los otros dos, en cada una de las computadoras. Ver figura 3. 


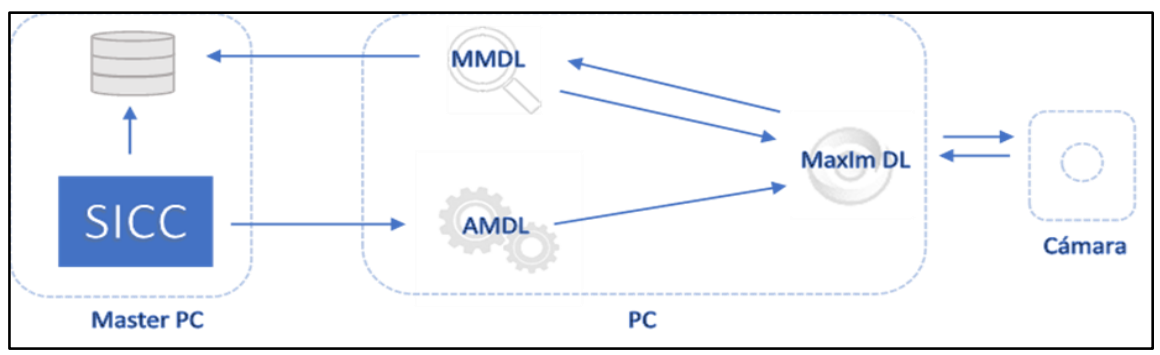

Fig. 3 En este diagrama se resume el flujo de información de SICC

El SICC en la Master PC envía órdenes de acciones a AMDL y ésta las ejecuta en Maxim DL que es el programa que se comunica directamente con la cámara. Por otro lado, MMDL monitorea los eventos de Maxim DL y el estatus de la cámara. Esta información es enviada a una base de datos en la Master PC. SICC monitorea la base de datos y muestra la información en la interfaz gráfica.

En cuanto a su funcionalidad, SICC se divide en cuatro secciones: General, que permite iniciar o cerrar el sistema, conectar o desconectar las cámaras, así como activar o desactivar su sistema de enfriamiento y activar el sistema de calentamiento seguro; Secuencia, que permite realizar una secuencia de imágenes simultaneas estableciendo la cantidad, tiempo de exposición, binning, etc. y muestra la información del estatus y acciones realizadas por las cámaras, así como el avance en las secuencias; Exposición, que permite realizar exposiciones individuales con las cámaras estableciendo el tiempo para luego mostrarlas en un visor; y $R D P$, que permite tener acceso a las computadoras a través de "Remote Desktop Connection" con un formato de pestañas.

Por otra parte, se han obtenido resultados preliminares alentadores que se aprecian en la figura 4. El equipo está listo para realizar observaciones prolongadas en la próxima estación seca. 


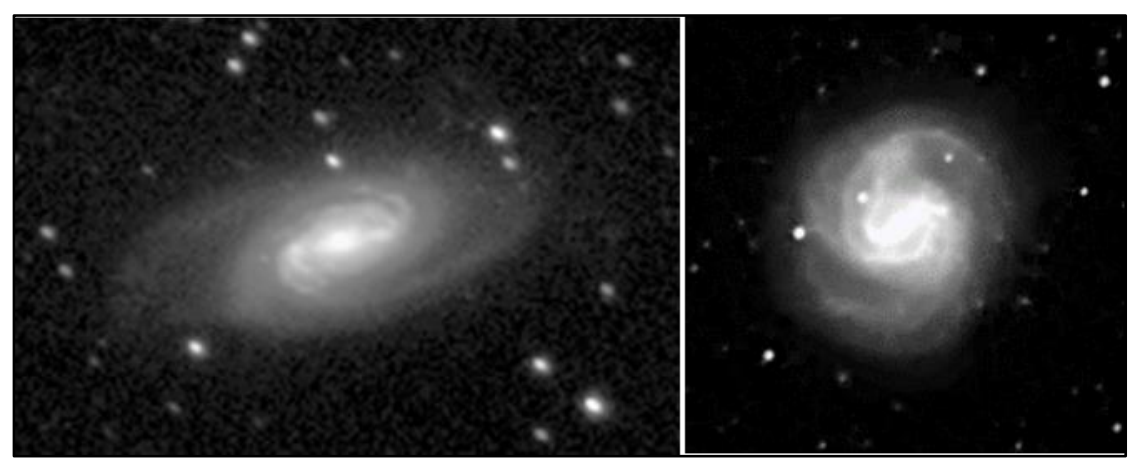

Fig. 4 Imágenes de las galaxias NGC9203 y M61 tomadas con SNORRI desde Churuquita Chiquita

En la figura 5 se presentan los resultados correspondientes a las pruebas de linealidad ("linearity"), ganancia ("gain") y ruido de lectura ("read-out noice") de una de las cámaras a temperatura de $-20^{\circ} \mathrm{C}$.

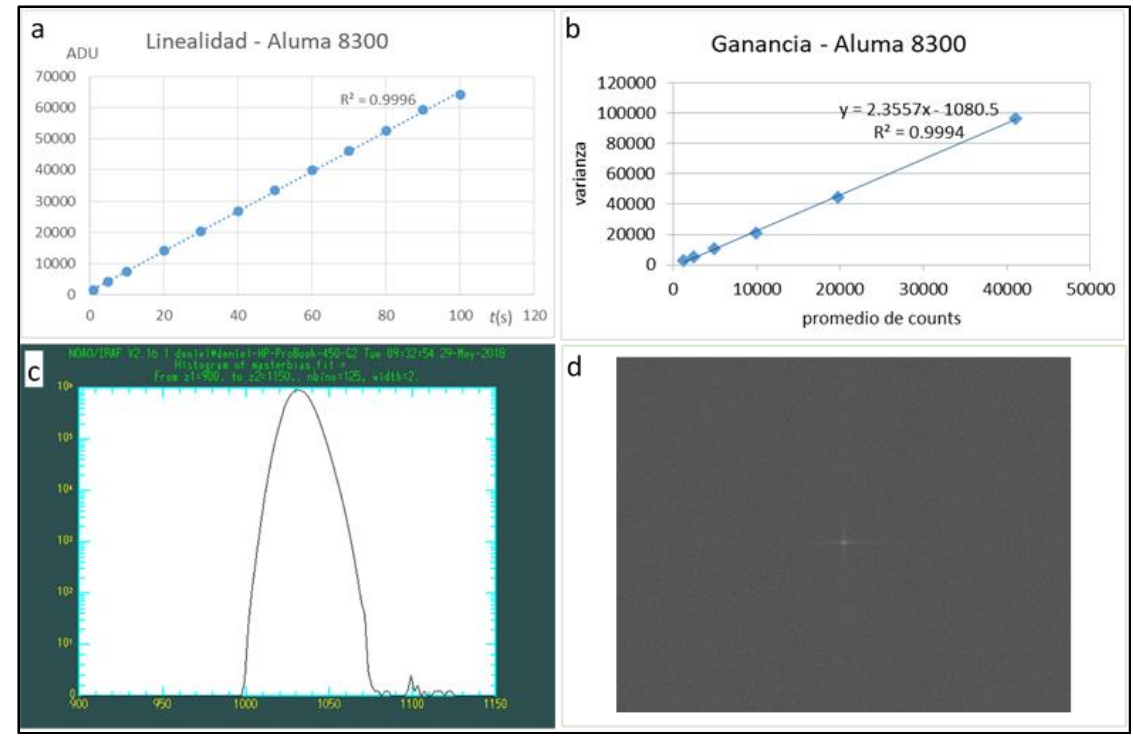

Fig. 5 (a) La cámara tiene una respuesta lineal muy buena que se refleja en el coeficiente de determinación. (b) Se obtuvo una ganancia o "gain" igual a $1 / 2.36=0.425$. (c) El ruido de lectura que se midió fue de 7.8, inferior al valor de 10 nominal de la Aluma 8300. (d) FFT del masterbias, 
En la inspección de calidad de la homogeneidad de los "masterbias" se pudo observar, usando Iraf (Tody, 1986), que el histograma se aproxima bastante a una gaussiana tendiendo a lo ideal. En la transformada de Fourier de la imagen o imagen FFT, se observa que existen patrones no aleatorios, aunque pequeños, que se perciben por la crucecita en el medio. Para las otras cámaras los resultados son similares.

Se realizó un análisis de la calidad de la imagen basada en un "light" sin calibrar con una exposición de 300 segundos. Los resultados se resumen en la figura 6 .

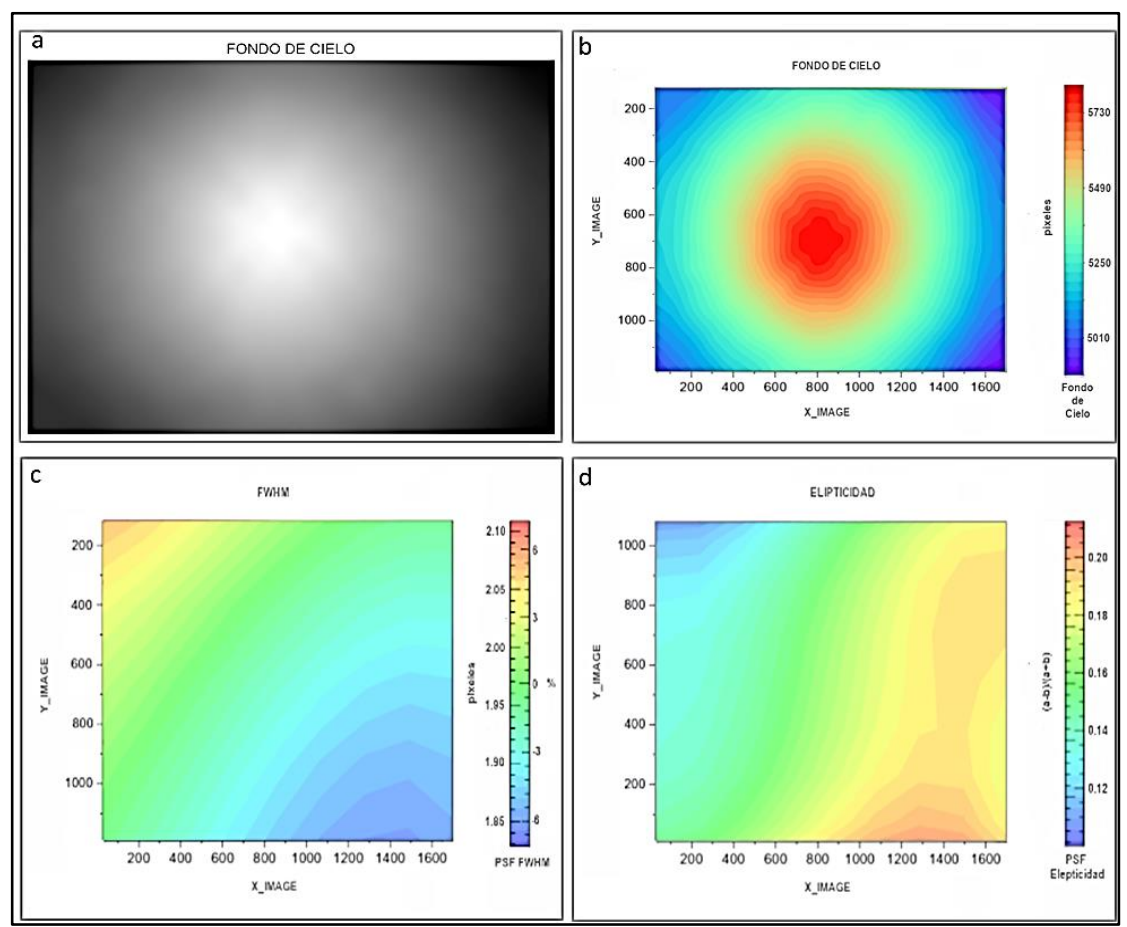

Fig. 6 (a) Fondo de cielo extraído de un "light" de 300s con SExtractor (Bertin \& Arnouts 1996) + PSFex (Bertin \& Arnouts 2017). (b) Mapa de contorno del fondo de cielo. (c) Mapa del FWHM. (d) Mapa de Elipticidad 
Como se puede ver en la imagen fondo de cielo (figura 6a), el patrón de iluminación está bien centrado en el chip, lo que indica una buena colimación general de los componentes ópticos en el lente. En el mapa de contorno de la derecha se puede observar que la iluminación cae aproximadamente un $15 \%$ desde el centro hasta el borde (figura $6 \mathrm{~b}$ ).

Hay un pequeño gradiente en los valores de la función de anchura a media altura (FWHM) (figura 6c). Las variaciones en FWHM son de aproximadamente $10 \%$, a ese nivel se ven comúnmente, y generalmente encuentran su origen en la precisión mecánica de la interfaz entre los lentes y las cámaras, así como una combinación de aberraciones intrínsecas en el diseño óptico de la lente e inclinación leve en el plano focal. El promedio de FWHM está por debajo de los 2 pixeles a lo largo de casi toda la imagen con una degradación máxima de $\sim 0.3$ píxeles. La elipticidad está por debajo del $18 \%$ a lo largo de casi toda la imagen (figura $6 \mathrm{~d}$ ).

La tabla 1 resume la estadística de los datos obtenidos de calidad de imagen.

Tabla 1 Calidad de imagen

\begin{tabular}{|c|c|c|c|c|c|c|c|c|}
\hline & $\begin{array}{c}\text { Objetos } \\
\text { Cargados }\end{array}$ & $\begin{array}{c}\text { Objetos } \\
\text { Aceptados }\end{array}$ & $\begin{array}{c}\text { Half-Light } \\
\text { diameter } \\
\text { (pix) }\end{array}$ & $\begin{array}{c}\text { FWHM } \\
\text { (pix) }\end{array}$ & Elipticidad & Residuos & Asimetría & $\begin{array}{c}\text { Área } \\
\text { Equivalente } \\
\text { de ruido }\end{array}$ \\
\hline Promedio & 1106 & 1046 & 2.01 & 1.91 & 0.15 & 0.02 & 0.06 & 0.04 \\
\hline Rango & $1106-1106$ & $1046-1046$ & $2.01-2.01$ & $1.86-2.07$ & $0.11-0.20$ & $0.02-0.04$ & $0.04-0.09$ & $0.03-0.07$ \\
\hline
\end{tabular}

Resultados comparables se han conseguido con equipo óptico similar (Abraham \& Van Dokkum, 2014), donde se obtuvo una variación en el decaimiento de la iluminación en la imagen fondo de cielo mayor, una degradación máxima en la FWHM levemente mayor, y una elipticidad menor. Los valores promedios menores en FWHM son de esperar, puesto que la calidad del cielo en ese lugar es mucho mejor que en zonas tropicales. La diferencia en los valores de elipticidad pueden ser atribuidos a la diferencia en los sistemas de autoguía. 


\section{CONCLUSIONES}

Este novedoso equipo de observación astronómica es único en la región por lo que se sabe. Debido al tipo de observaciones que se pretenden realizar, se requiere de tiempo y paciencia. Por la calidad del equipo y las pruebas que se han hecho se puede afirmar que se cuenta con un instrumento inédito que marca un antes y un después en el desarrollo de la astronomía y la astrofísica en la República de Panamá.

\section{AGRADECIMIENTOS}

A todos los involucrados en la gestión y coordinación de este proyecto por parte de la Secretaría Nacional de Ciencia, Tecnología e Innovación (SENACYT) de la República de Panamá.

\section{REFERENCIAS}

Abraham, R. \& Van Dokkum, P. 2014. Ultra-Low Surface Brightness Imaging with the Dragonfly Telephoto Array. Publ. Astron. Soc. Pac. 126(935): 55-69

Bertin, E. \& Arnouts, S. 1996. SExtractor: Software for source extraction. Astron. Astrophys. Suppl. 117: 393-404.

Bertin, E., Moneti A. 2017. PSFEx Documentation: Release 3.18.2. PSF Extractor. Retrieved from https://buildmedia.readthedocs.org/ media/pdf/psfex/latest/psfex.pdf on October 6, 2019

Bullock, J. \& Johnston, K. 2005. Tracing galaxy formation with stellar halos I: Methods. arXiv:astro-ph/0506467v1.

Canon Inc. 2011. Canon EF Lens EF400mm f/2.8L IS II USM: instruction manual. Retrieved from https://www.usa.canon.com/ internet/portal/us/home/support/details/lenses/ef/super-telephoto/ef-

400mm-f-2-81-is-ii-usm?tab=manuals on October 9, 2019

Cooper A. P et al: Galactic stellar haloes in the CDM model. 2010. Royal Astronomical Society. Volume 406, Issue 2, pp. 744-766. 
Diffraction Limited. 2019. Aluma: User's Manual. Retrieved from http://diffractionlimited.com/downloads/AlumaUsersManual.pdf on October 15, 2019

Diffraction Limited. 2019. Cyanogen Imaging ${ }^{\circledR}$ MaxIm DL: User's Manual. Retrieved from http://diffractionlimited.com/help/ maximdl/MaxIm-DL.htm on October 15, 2019

Martínez-Delgado D. et al. 2010. Stellar tidal streams in spiral galaxies of the local volume: a pilot survey with modest aperture telescopes; AJ 140, 962-967.

Martínez-Delgado D. et al. 2015). A Stellar Tidal Stream Around the Whale Galaxy, NGC 4631; AJ 150, 116.

Software Bisque. 2015. The Paramount Robotic Telescope System: User Guide. Retrieved from http://w.bisque.com/sc/media/p/ 75568.aspx on October 15, 2019

Software Bisque. 2019. TheSkyX Professional and Serious Astronomer Edition: User Guide. Retrieved from http://w.bisque.com/sc/media/p/ 30354.aspx on October 15, 2019

Van Dokkum, P., Abraham, R. \& Merritt, A. 2014. First results from the Dragonfly Telephoto Array: The Apparent Lack of Stellar Halo in the Massive Spiral Galaxy M101; AJ 782, 124.

Tody, D. 1986. The IRAF data reduction and analysis system. In Proc. SPIE. Instrumentation and Astronomy VI, Ed. D.L. Crawford, 627, 733

Recibido 8 de octubre de 2019, aceptado 20 de noviembre de 2019. 